hashtag created to disseminate Gynaecological Oncology specific information from the American Society of Clinical Oncology (ASCO) 2020 Virtual Annual Meeting 29th May to 1st June 2020.

Methodology Prospective observational study of tweets/retweets associated with \#goASCO20. All tweets using \#goASCO20/ \#ASCO20 during ASCO 2020 were extracted using NodeXL. Data was analysed in Excel identifying individual tweets, tweeters, retweets and retweeters. Analysing original tweets (replies excluded) \#goASCO20 was compared to \#ASCO20 (official hashtag), \#gyncsm and \#SGOatASCO.

Results 1,811 tweeters used \#goASCO20/\#ASCO20 in 11,530 tweets. These tweets received 27,962 retweets. $12.4 \%$ (224) of tweeters received $80 \%$ of all retweets, while $34.2 \%$ (619) received no retweets. 7,644 accounts tweeted/retweeted using \#ASCO20 hashtag, producing 11,455 tweets and 27,888 retweets. $11.1 \%$ of accounts (850) only tweeted, $76.3 \%$ $(5,833)$ just retweeted, and $12.6 \%$ (961) both tweeted and retweeted.

\#goASCO20 was used in 200 tweets and generated 262 retweets. This activity stemmed from 38 accounts that tweeted/retweeted using \#goASCO20. Of these accounts 13\% (5) just tweeted, 74\% (28) just retweeted and 13\% (5) both tweeted and retweeted.

Top hashtags used during ASCO 2020 are shown in table 1. Figure 1 compares Gynaecology related hashtags used during the conference. \#goASCO20 hashtag was number 13 by tweets and 27th by retweets during the conference period.

Conclusion '\#goASCO20', a unique Gynaecological Oncology hashtag was created to disseminate Gynaecological Oncology information from ASCO 2020 virtual event. \#goASCO20 was one of several gynaecological oncology hashtags used. Each of these were used by relatively small groups of individuals. Discussions were fragmented resulting in an overall lower profile for Gynaecological Oncology related tweets compared to general tweeting during ASCO 2020. The use of Twitter in academia is increasing. Its use during such conferences facilitates the spread of clinical knowledge; arguably more than any other academic platform. Gynaecological oncology tweeting needs coordination and agreement on a common hashtag to organise content at virtual events and between meetings.

Disclosures Esra Bilir: Member of Communication and Social Media Committee at The European Network of Young Gynae Oncologists (ENYGO) since May 2020.

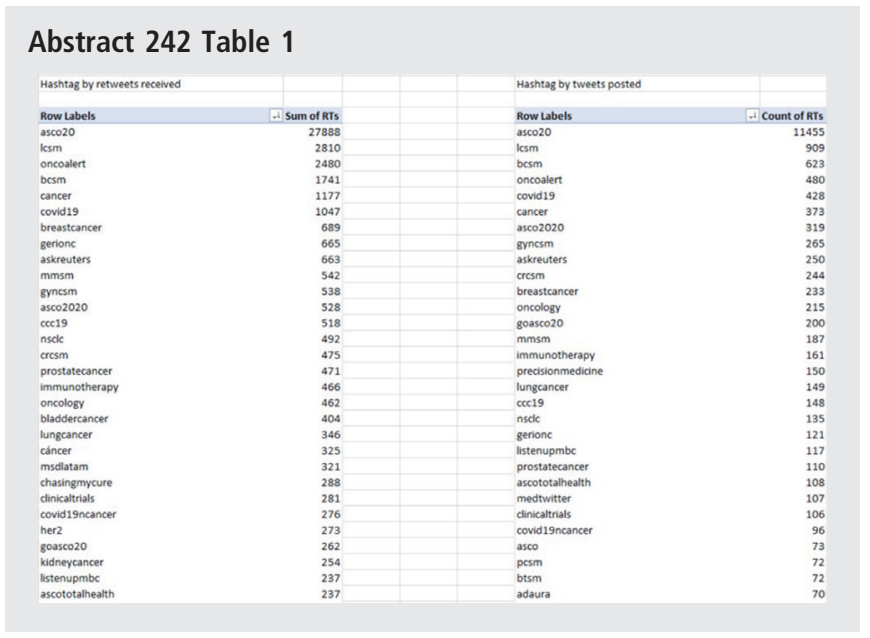

Christina Uwins: Nothing to declare

Graham Mackenzie: Nothing to declare.

İlker Selçuk: European Network of Young Gynae-Oncologists (ENYGO) Executive Group Member (2017-2021) (ENYGO Social Media Committee Chair)

Geetu Bhandoria: Nothing to declare

\section{GYNAECOLOGIC AND NON-GYNAECOLOGIC TUMORS METASTATIC TO THE OVARIES}

Gunel Ziyadova, Mehmet Coskun Salman, Murat Gultekin, Nejat Ozgul. Hacettepe University Faculty of Medicine; Department of Obstetrics and Gynaecology

\subsection{6/ijgc-2020-ESG0.92}

Introduction/Background The ovaries are a frequent site of metastasis and tumors metastatic to the ovaries account for 5$6 \%$ of all ovarian tumors. In most cases, the primary tumor originates from gastrointestinal tract, breast, and gynaecologic organs including endometrium and cervix. The diagnosis is often made on final pathology since many metastatic tumors mimics primary ovarian carcinoma and most patients present with pelvic mass. Therefore, surgery is the mainstay of management. The prognosis of patients with ovarian metastasis is generally poor. However, survival rates are even worse in patients with non-gynaecologic primaries.

Methodology Patients with pelvic mass who were operated at Hacettepe University Faculty of Medicine, Department of Obstetrics and Gynaecology for a five-year period were identified. Among them, the clinical and pathological characteristics of patients with tumors metastatic to the ovaries were retrospectively reviewed. Survival analysis was done as well with a particular focus on the origin of the primary tumor.

Results Tumors metastatic to the ovaries accounted for $16.2 \%$ of all ovarian malignancies and 79 cases with ovarian metastases constituted the study group. Mean age of the patients was 56.3 years. Primary tumor non-gynaecologic in $65.8 \%$ of cases and colon cancer was the most common non-gynaecologic primary followed by stomach and breast cancer. All remaining patients $(34.2 \%)$ had primary endometrial cancer metastatic to the ovaries. Patients with gynaecologic primaries were significantly older, but the levels of tumor markers were similar. Patients with non-gynaecologic primaries mostcommonly presented with abnormal imaging results and pain while abnormal bleeding was the most common symptom in those with gynaecologic primaries. Staging surgery and total abdominal hysterectomy with bilateral salpingo-oophorectomy were the most common surgeries performed. Bilateral ovarian involvement was detected in $62.0 \%$ of cases. Mean diameter of the tumor was $6.5 \mathrm{~cm}$. Adjuvant treatment was given in $96.2 \%$ of patients. Of patients, $43.0 \%$ died of disease. Median survival was 19.7 months, but this was significantly longer in patients with non-gynaecologic primaries (10.0 months vs 32.6 months, $\mathrm{p}=0.05)$. Longest survival was detected in patients with colon cancer. Extent of surgery or the type of adjuvant therapy given did not affect median survival.

Conclusion Tumors metastatic to the ovaries account for a significant proportion of all ovarian cancers. Most of these tumors are diagnosed in elderly patients and prognosis is 
relatively poor regardless of the surgical procedures performed and adjuvant therapies given.

Disclosures No potential conflict of interest to declare.

\section{A SYSTEMATIC REVIEW TO IDENTIFY AND ASSESS THE MENTAL HEALTH SEQUALAE AMONGST WOMEN WITH ENDOMETRIOSIS WITH OR WITHOUT CHRONIC PELVIC PAIN (THE ELEMI PROJECT)}

'Dharani Hapangama, ${ }^{2}$ Gayathri Delanerolle, ${ }^{3}$ Peter Phiri, ${ }^{2}$ Rema Ramakrishnan, ${ }^{4}$ Ashish Shetty, ${ }^{5}$ Trusha Kotari, ${ }^{6}$ Katherine Barnard, ${ }^{7}$ Molola Oyewole, ${ }^{8}$ Damien Longson, ${ }^{9}$ Vanessa Raymont. 'University of Liverpool; Liverpool Women's Hospital NHS Trust; ${ }^{2}$ University of Oxford; ${ }^{3}$ Southern Health NHS Trust; University of Southampton; ${ }^{4}$ University College London NHS Foundation Trust; University College London; ${ }^{5}$ Liverpool Women's Hospital NHS Trust; ${ }^{6}$ University of Southampton; 'University College London NHS Foundation Trust; ${ }^{8}$ University of Manchester; ${ }^{9}$ University of Oxford; Oxford Health NHS Foundation Trust

\subsection{6/ijgc-2020-ESG0.93}

Introduction/Background Endometriosis is a complex, chronic gynaecological condition impacting approximately 176 million women globally. It is associated with symptoms such as chronic pelvic pain (CPP), dysmenorrhoea, menorrhagia, sexual dysfunction and infertility. During several points in the lifecycle of this chronic disease, women bear the consequent burden of mental health $(\mathrm{MH})$ difficulties, due to the complex symptomatology and comorbidities of endometriosis. For example, delayed diagnosis (the average time to diagnose being 7-8 years), undergoing often repeated

\section{Forest plot of pooled prevalence of anxiety}

\begin{tabular}{lll} 
Author & $\begin{array}{l}\text { Percent } \\
(95 \% \mathrm{Cl})\end{array}$ \\
\hline Cavaggioni, 2014 & $30.00(20.26,41.28)$ \\
De Graaff, 2016 & $32.51(26.66,38.79)$ \\
Friedl, 2015 & $17.74(9.20,29.53)$ \\
Laganà, 2015 & $40.96(33.40,48.85)$ \\
Sepulcri, 2009 & $28.85(20.38,38.55)$ \\
Simoens, 2012 & $35.97(32.85,39.19)$ \\
Overall (I $=72.7 \%, \mathrm{p}<0.001)$ & $31.80(26.51,37.09)$ \\
& 20 & 100 \\
\hline A & Percent
\end{tabular}

Forest plot of pooled prevalence of depression

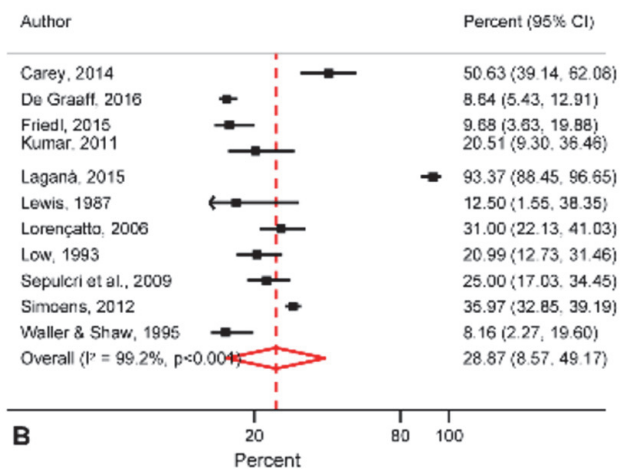

Abstract 369 Figure 1 excision surgeries, and difficulties with subfertility and sexual activities, and suffering with long-term CPP and analgesic use, can all negatively influence $\mathrm{MH}$ and can have significant impact on the psychological, sexual, relationship and social functioning of affected women. Therefore, a systematic review was conducted as part of the evidence synthesis phase of the ELEMI project to identify and assess this complex relationship with a view to report on any knowledge and clinical practice gaps.

Methodology The systematic protocol was published in PROSPERO (CRD42020181495). MeSH terms developed include Endometriosis, Depression, Anxiety, Low mood, Psychiatry comorbidity, Women's health and CPP. All studies and material published between January 1980 to June 2020 in English and participants were included.

Results Out of 28 studies included in the systematic review, 17 were included in the meta-analysis (anxiety: 6, chronic pelvic pain: 3, depression: 11, and dyspareunia: 3) which described the prevalence and extent of $\mathrm{MH}$ symptoms in endometriosis and/or CPP. The pooled prevalence for anxiety was found to be $31.8 \%$ (95\% CI: $26.5 \%$ - 37.1\%), whilst for depression 28.9\% (95\%CI: 8.6\%-49.2\%). Pooled prevalence for CPP was high at 57.2\% (95\%CI: $7.0 \%$, $107.4 \%)$ and pooled estimate of mean SF-MPQ for chronic pain to be 13.09 (95\%CI: 7.13, 19.05). Computed prevalence for dyspareunia was also identified to be high (prevalence: $54.9 \%$, 95\% CI: 43.9\%, 65.9\%). The narrative analysis showed depression and anxiety to be the most commonly reported $\mathrm{MH}$ symptoms. None of the papers indicated if these had received a clinical diagnosis or were

\section{Forest plot of pooled prevalence of chronic pelvic pain}

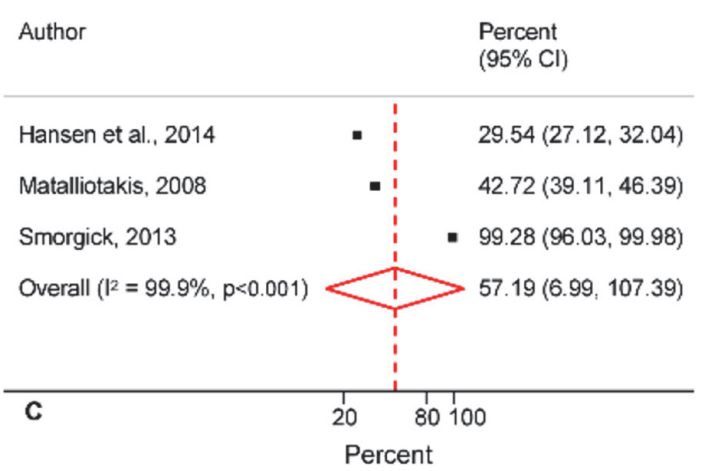

Forest plot of pooled prevalence of dyspareunia

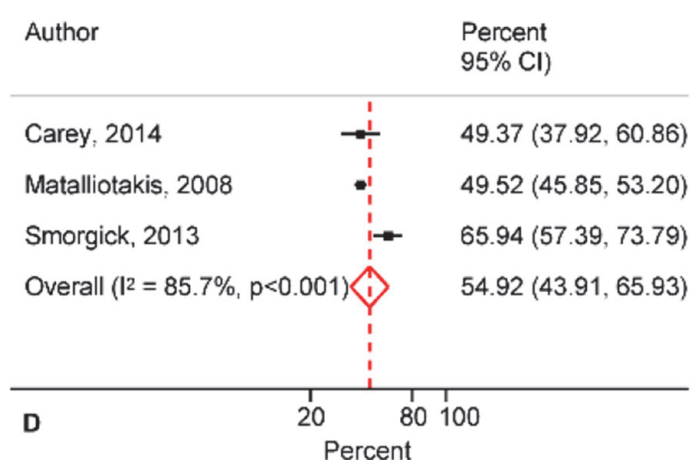

